\title{
Crystallographic orientation of facets and planar defects in functional nanostructures elucidated by nano-focused coherent diffractive X-ray imaging
}

Citation for published version (APA):

Richard, M. I., Fernández, S., Eymery, J., Hofmann, J. P., Gao, L., Carnis, J., Labat, S., Favre-Nicolin, V., Hensen, E. J.' M., Thomas, O., Schülli, T. U., \& Leake, S. J. (2018). Crystallographic orientation of facets and planar defects in functional nanostructures elucidated by nano-focused coherent diffractive X-ray imaging. Nanoscale, 10(10), 4833-4840. https://doi.org/10.1039/C7NR07990G

DOI:

10.1039/C7NR07990G

Document status and date:

Published: 14/03/2018

\section{Document Version:}

Accepted manuscript including changes made at the peer-review stage

\section{Please check the document version of this publication:}

- A submitted manuscript is the version of the article upon submission and before peer-review. There can be important differences between the submitted version and the official published version of record. People interested in the research are advised to contact the author for the final version of the publication, or visit the DOI to the publisher's website.

- The final author version and the galley proof are versions of the publication after peer review.

- The final published version features the final layout of the paper including the volume, issue and page numbers.

Link to publication

\footnotetext{
General rights

- You may freely distribute the URL identifying the publication in the public portal. follow below link for the End User Agreement:

www.tue.nl/taverne

\section{Take down policy}

If you believe that this document breaches copyright please contact us at:

openaccess@tue.nl

providing details and we will investigate your claim.
}

Copyright and moral rights for the publications made accessible in the public portal are retained by the authors and/or other copyright owners and it is a condition of accessing publications that users recognise and abide by the legal requirements associated with these rights.

- Users may download and print one copy of any publication from the public portal for the purpose of private study or research.

- You may not further distribute the material or use it for any profit-making activity or commercial gain

If the publication is distributed under the terms of Article $25 \mathrm{fa}$ of the Dutch Copyright Act, indicated by the "Taverne" license above, please 

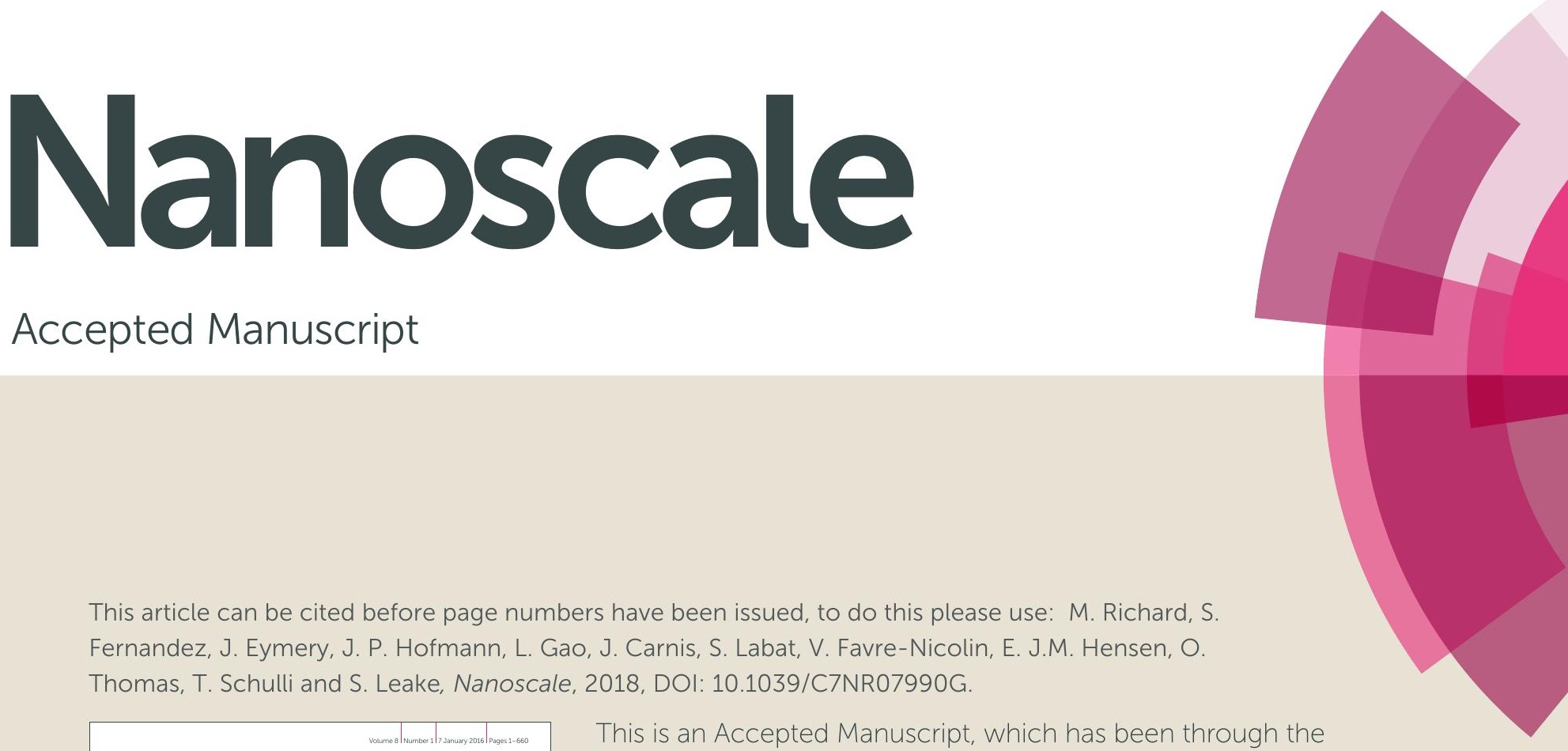

\section{Accepted Manuscript}

This article can be cited before page numbers have been issued, to do this please use: M. Richard, S. Fernandez, J. Eymery, J. P. Hofmann, L. Gao, J. Carnis, S. Labat, V. Favre-Nicolin, E. J.M. Hensen, O. Thomas, T. Schulli and S. Leake, Nanoscale, 2018, DOI: 10.1039/C7NR07990G.

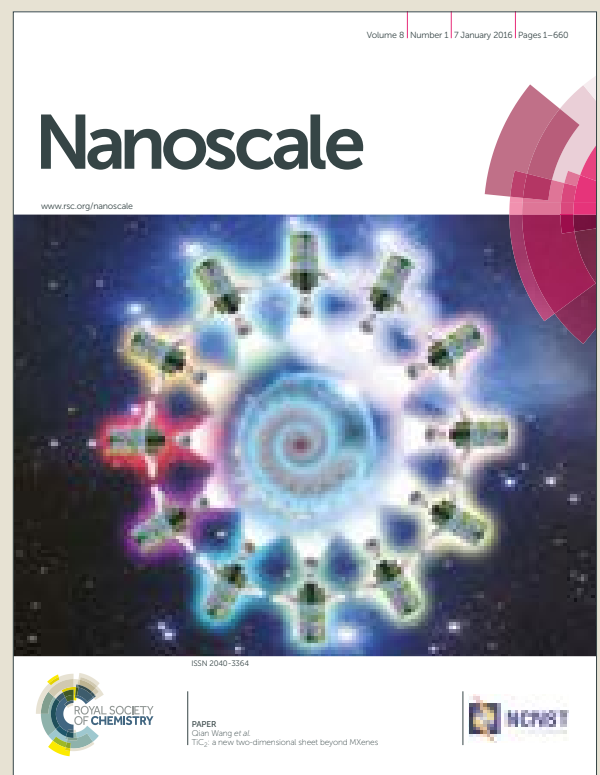

This is an Accepted Manuscript, which has been through the Royal Society of Chemistry peer review process and has been accepted for publication.

Accepted Manuscripts are published online shortly after acceptance, before technical editing, formatting and proof reading. Using this free service, authors can make their results available to the community, in citable form, before we publish the edited article. We will replace this Accepted Manuscript with the edited and formatted Advance Article as soon as it is available.

You can find more information about Accepted Manuscripts in the author guidelines.

Please note that technical editing may introduce minor changes to the text and/or graphics, which may alter content. The journal's standard Terms \& Conditions and the ethical guidelines, outlined in our author and reviewer resource centre, still apply. In no event shall the Royal Society of Chemistry be held responsible for any errors or omissions in this Accepted Manuscript or any consequences arising from the use of any information it contains. 


\title{
Journal Name
}

\section{ARTICLE TYPE}

Cite this: DOI: $10.1039 /$ xxxxxxxxxx

\section{Crystallographic orientation of facets and planar de- fects in functional nanostructures elucidated by nano- focused coherent diffractive x-ray imaging}

\author{
Marie-Ingrid Richard, ${ }^{* a, b}$, Sara Fernández ${ }^{a, b}$, Joël Eymery ${ }^{c, d}$, Jan Philipp Hofmann ${ }^{e}$, Lu \\ $\mathrm{Gao}^{e}$, Jérôme Carnis ${ }^{a, b}$, Stéphane Labat ${ }^{a}$, Vincent Favre-Nicolin ${ }^{b}$, Emiel J. M. Hensen ${ }^{e}$, \\ Olivier Thomas ${ }^{a}$, T. Schülli ${ }^{b}$, S. Leake ${ }^{b}$
}

Received Date
Accepted Date

DOI: $10.1039 / x x x x x x x x x x$

www.rsc.org/journalname

\begin{abstract}
The physical and chemical properties of nanostructures depend on their surface facets. Here, we exploit a pole figure approach to determine the three-dimensional orientation matrix of a nanostructure from a single Bragg reflection measured with a coherent nano-focused $x$-ray beam. The signature of any truncated (faceted) crystal produces a crystal truncation rod, which corresponds to a streak of intensity in reciprocal space normal to the surface. When two or more non-parallel facets are present, both the crystal orientation and the crystal facets can be identified. This enables facets to be rapidly indexed and uncommon facets, and planar defects, that have been difficult to study before to be identified. We demonstrate the technique with (i) epitaxial coreshell InGaN/GaN multiple quantum-wells grown on GaN nanowires, where surface facets and planar defects are determined, and (ii) single randomly oriented highly faceted tetrahedrahexal Pt nanoparticles. The methodology is applicable to a broad range of nanocrystals and provides a unique insight into the connection between structure and properties of nanomaterials.
\end{abstract}

\section{Introduction}

Better understanding of nanocrystal growth and systematic control of morphologies are crucial for uncovering many shapedependent physical and chemical properties, for instance catalytic $^{1}$, plasmonic ${ }^{2}$ and optical characteristics. The physical properties of nanostructures depend on the type of surface facets, which have a strong influence on crucial effects such as reactivity or adsorption. For instance the growth rate of semiconductors is affected by facet orientation. For non-centrosymmetric structures, it also changes the polarisation fields (spontaneous and piezoelectric) applied to quantum dots (QDs) or multiple quantum wells (MQWs). Faceting often results from minimisation of surface energy as described by J. W. Gibbs in the $1870 \mathrm{~s}^{3}$ : during crystal growth, high-energy facets will grow at a higher rate than low-energy facets and eventually disappear, resulting in a

\footnotetext{
${ }^{a}$ Aix Marseille Université, CNRS, Université de Toulon, IM2NP UMR 7334, 13397, Marseille, France; E-mail: marie-ingrid.richard@im2np.fr

${ }^{b}$ ESRF - The European Synchrotron, 71 Ave. des Martyrs, 38000 Grenoble, France.

${ }^{c}$ Univ. Grenoble Alpes, F-38000 Grenoble, France.

${ }^{d}$ CEA, INAC-MEM, Nanostructures and Synchrotron Radiation Laboratory, F-38000 Grenoble, France.

${ }^{e}$ Laboratory of Inorganic Materials Chemistry, Department of Chemical Engineering and Chemistry, Eindhoven University of Technology, P. O. Box 513, 5600MB Eindhoven, The Netherlands.
}

nanocrystal whose shape is adjusted to minimise surface energy. Several growth methods such as Metal-Organic Vapour Phase Epitaxy (MOVPE) are far from thermodynamical equilibrium and are mainly driven by kinetics and facets are hardly predictable and depend tremendously on the growth process. Understanding how facets develop on a nanocrystal is essential to controlling the crystal's geometric shape, which in turn is critical to controlling the crystal's chemical and electronic properties. A thorough understanding of the formation of facets at the surface is required to optimise their properties. The study of nanocrystal shape evolution is a poignant topic in the field of nanomaterials when it comes to rationally engineer nanocrystals with desirable surface structures, and hence properties.

Morphologies of nanocrystals can be characterised by highresolution transmission electron microscopy (HRTEM). Significant progress has recently been achieved pushing the resolution to the atomic level using aberration-corrected electron lenses ${ }^{4}$ or high-angle annular dark-field scanning transmission electron microscopy (HAADF-STEM) ${ }^{516}$, where the surface facets can be characterised with a high accuracy. Recently, coherent electron diffraction ${ }^{7}$ has also been used to characterise high-index facets in nanocrystals as large as $\sim 100 \mathrm{~nm}$. However, for larger particles this is more challenging. Surface morphologies can also 
be determined in three dimensions (3D) using electron tomography. ${ }^{8}$ However, the resolution is often unsatisfactory to allow a straightforward characterisation of the crystallographic planes at the surface, especially when considering high-index facets. Applications to nanoparticles often show rounded facets, which are insufficiently resolved for the determination of high-index facets. In all cases, microscopy requires orienting the sample to have the beam parallel to the various facets to be indexed. Therefore, other approaches should be explored that enable a direct identification of all surface facets.

Coherent x-ray diffraction imaging (CXDI) is a lensless imaging technique whereby the intensity local to each Bragg peak encodes the internal displacement field of the illuminated object ${ }^{9}$, when sufficient oversampling is used. Identifying a single Bragg reflection is often straightforward, if the crystal growth axis is known. However the technical challenge of rotating a crystal through many axes to measure additional Bragg peaks is significant and often requires additional sample characterisation in the form of scanning electron microscopy (SEM), confocal microscopy, electron backscatter diffraction (EBSD) analysis 10 or micro-Laue diffraction 11. As CXDI matures towards in situ and operando experiments, pre-characterisation becomes unfeasible.

Here, we demonstrate a method to identify the facets present on a crystal providing a fast on-the-fly method to monitor facet modulation. In addition, one can determine the orientation matrix to sufficient accuracy to easily locate and measure multiple Bragg reflections from the same object. A three-dimensional (3D) data set at a given hkl Bragg reflection is measured on a single nanostructure using a nano-focused x-ray beam. From the stereographic projection or pole figure of this 3D data set, the surface facets and planar defects can be characterised and indexed. Contrary to microscopy, the sample is aligned once: one single rocking-curve (few degrees) can give the crystal orientation. The method allows a straightforward characterisation of the crystallographic planes at the surface, which can be for instance compared with the Wulff construction. We have applied this technique to two types of nanostructure: (1) epitaxial core-shell InGaN/GaN multiple quantum-wells grown on GaN nanowires and (2) randomly oriented single high index faceted platinum (Pt) particles. InGaN ternary alloys are of great importance due to their potential to provide tunable emission over most of the solar spectrum using one single crystalline material by simply adjusting the alloy composition. ${ }^{12}$ The nanostructure geometry can have a significant impact on both light extraction and collection efficiencies. $\frac{13}{13}$ InGaN ternary alloys incorporated in multiple quantum well barriers also exhibit competitive emission and absorption properties $\frac{14115}{15}$ For this example, the crystallographic orientation of the side facets as well as planar defects along a single coreshell InGaN/GaN multiple quantum-wells on a GaN nanowire have been determined in a straightforward way. Regarding the second showcase, platinum is one of the most effective industrial catalysts in use today. The pioneering work of Sun et al. has resulted in the controlled syntheses of regularly shaped Pt particles as well as alloys of Pt group metals (PGMs) with highindex facets. $\frac{16] 17}{10}$ In contrast to close-packed low-index faceted single crystals, nanoparticles with atomically open (high-index) surfaces are often more active - and even more active than conventional nanoparticles. ${ }^{18}$ Atomic steps, edges, and kinks usually serve as active sites for breaking chemical bonds. Determining how facets evolve during catalysis is highly desirable to set-up structure-activity, structure-selectivity and structure-stability relationships in heterogeneous catalytic systems.

\section{Experimental Section}

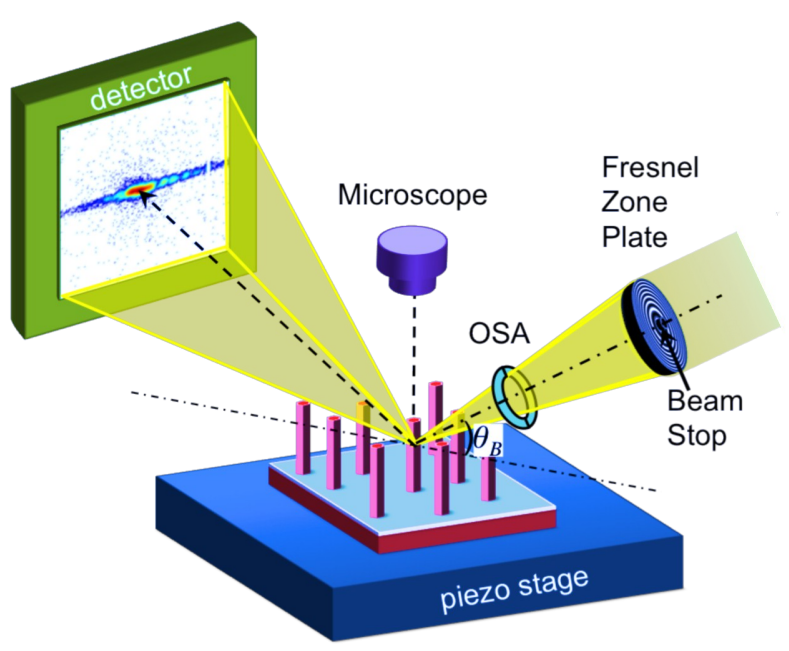

Fig. 1 Schematic representation of the experimental set-up in co-planar diffraction geometry. The incoming $x$-ray beam is focused on an identified single nanostructure using a Fresnel Zone-Plate and an order sorting aperture (OSA). The Bragg angle $\theta_{B}$ is displayed.

The first sample consists of self-assembled GaN wires obtained by organometallic vapor phase epitaxy on $c$-sapphire substrates without a catalyst. ${ }^{14}$ InGaN/GaN multiple quantum wells (quantum well thickness of $5 \mathrm{~nm}$ ) have been then grown on the nonpolar $\{1-100\}$ sidewalls of c-axis GaN wires. The MOCVD deposition of InGaN layers is performed under nitrogen at 400 mbar using triethylgallium (TEG) and trimethylindium (TMI) precursors. After the growth of the $\mathrm{GaN}$ core at high temperature (around $1000^{\circ} \mathrm{C}$ ), the temperature is decreased to about $720-760^{\circ} \mathrm{C}$ to grow InGaN and then rapidly increased to $830-$ $870^{\circ} \mathrm{C}$ to deposit the GaN barrier, again using the TEG precursor. This temperature is chosen as a compromise between achieving a high quality GaN barrier and preventing indium desorption/interdiffusion in the well. 14 The average length and diameter of the nanowires are $25 \mu \mathrm{m}$ and $\sim 500-800 \mathrm{~nm}$, respectively ${ }^{[14] 15}$ The efficiency of these wires has been demonstrated for electroluminescent devices. ${ }^{14}$ They also show a conventional epitaxial relationship with sapphire corresponding to a $30^{\circ}$ rotation of the two unit cells along the $c$-axis: $[1 \overline{1} 00]_{\text {GaN }} / /[1 \overline{2} 10]_{\text {Sapp }}$ and $[0001]_{\text {GaN }} / /[0001]_{\text {Sapp }}, 19$ which is very convenient to align the wires.

The second sample consists of randomly oriented highindex tetrahexahedral (THH) pure or sub-surface $\mathrm{Cu}$ alloyed $\mathrm{Pt}$ nanocrystals. They have been synthesised on glassy carbon (GC) electrodes by a square-wave-potential method with a saturated calomel reference electrode (SCE) and a Pt foil counter electrode in $2 \mathrm{mM} \mathrm{H}_{2} \mathrm{PtCl}_{6}$ and $0.1 \mathrm{M} \mathrm{H}_{2} \mathrm{SO}_{4}$ electrolyte following the pro- 


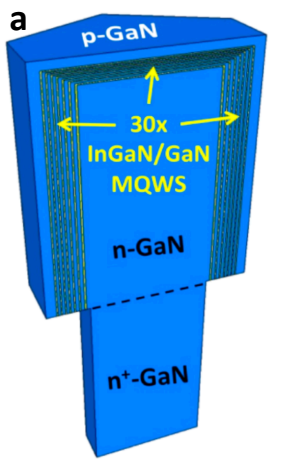

b

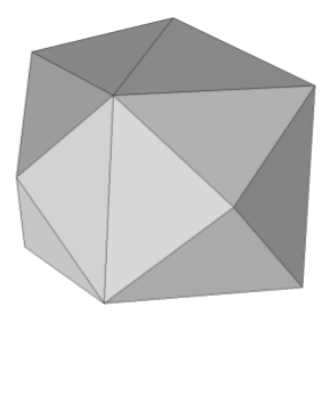

Fig. 2 Schematic overview of the two samples: (a) core-shell InGaN/GaN multiple quantum wells (MQWs) nanowire and (b) high-index tetrahexahedral Pt nanocrystal.

cedure described in Ref. 16 . The GC electrode is first subjected to a potential of $+1.20 \mathrm{~V}$ for $2 \mathrm{~s}$ to clean the surface and then -0.35 $\mathrm{V}$ for $60 \mathrm{~ms}$ to create Pt nuclei. The Pt nuclei grew into THH particles by applying a square-wave-potential between $+0.04 \mathrm{~V}$ and $+1.09 \mathrm{~V}$ at $100 \mathrm{~Hz}$ for $10 \mathrm{~min}$. The THH shape can be described as a cube whose faces are capped by square-based pyramids, leading to 24 high-index planes of $h k 0$ type $(h \neq k \neq 0) .20$ The diameter of the Pt NCs varies from 50 to $600 \mathrm{~nm}$.

Nano-focused x-ray measurements were performed at the ID01 beamline of the European Synchrotron Radiation Facility (see Fig. 1). Diffraction was measured by a two-dimensional (2D) pixel MAXIPIX detector 21 (516 x 516 pixels of $55 \mu \mathrm{m}$ placed at a distance of $1.11 \mathrm{~m}$ from the sample). A coherent portion of the monochromatic ( $9.0 \mathrm{keV}$, wavelength $\lambda$ of $1.378 \AA$ ) beam was selected with high precision slits by matching their horizontal and vertical gaps with the transverse coherence lengths of the beamline $(60 \mu \mathrm{m}$ (horizontally) and $200 \mu \mathrm{m}$ (vertically) close to the sample position). The coherent beam was then focused to 700 nm x $400 \mathrm{~nm}$ using a Fresnel Zone Plate (diameter of $300 \mu \mathrm{m}$ ) or Kirkpatrick-Baez (KB) mirrors, in order to illuminate a single nanowire or nanoparticle. Three dimensional data sets were recorded by rocking the sample over a range of $1.4^{\circ}$ in steps of $0.0047^{\circ}$ for the measured nanowires and over a range of $2.6^{\circ}$ in steps of $0.02^{\circ}$ for the Pt THH particles.

\section{Results and Discussion}

\subsection{Case of InGaN/GaN multiple quantum wells grown on the sidewalls of c-axis GaN wires.}

Knowing that the wires have the crystallographic [00-1] direction along the long axis (as wires are N-polar, but the minus sign will be omitted in this paper to simplify the notations), the measurement of the out-of-plane $\mathbf{0 0 4} \mathrm{GaN}$ reflection is straightforward: the incident angle of the sample and the out-of-plane angle of the detector are fixed to $\theta_{B}$ and $2 \theta_{B}$, respectively, where $\theta_{B r a g g}$ corresponds to the Bragg angle of the $004 \mathrm{GaN}$ reflection. Figures 3(a-c) display 3D reciprocal space maps around the $004 \mathrm{GaN}$ Bragg reflection for two different single GaN nanowires (with InGaN/GaN multiple quantum wells grown on the sidewalls) and for the second nanowire at two different $z$ positions along the nanowire. The $004 \mathrm{GaN}$ reflection was measured at a Bragg an- gle of $\theta_{B}=32.15^{\circ}$. Streaked Bragg peaks are observed: around each of the Bragg reflections, we observe at least twelve strong streaks. The conversion of the $2 \mathrm{D}$ detector images into $3 \mathrm{D}$ reciprocal space maps of reciprocal space coordinates $\left(Q_{x}, Q_{y}\right.$ and $Q_{z}$, where $Q=4 \pi / \lambda \sin (\theta)$ ) has been performed using the xrayutilities Python package (http://xrayutilities.sourceforge.net).22

In the kinematical approximation, the scattered intensity is proportional to the squared modulus of the Fourier transform of the complex-valued real-space object $\tilde{\Omega}(\vec{r})$, which satisfies:

$$
\tilde{\Omega}(\vec{r})=\rho_{e}(\vec{r}) \cdot e^{i \vec{Q} \cdot \vec{u}(\vec{r})}
$$

where $\vec{Q}$ is the reciprocal space lattice vector as mentioned above, $\rho_{e}(\vec{r})$ is the electronic density of the crystal (the particle shape $S(\vec{r})$ is enclosed in $\left.\rho_{e}(\vec{r})\right)$ and $\vec{u}(\vec{r})$ is the atomic displacement field. For faceted crystals, reciprocal lattice points transform into shape functions consisting of reciprocal rods (or streaks) perpendicular to each facet. The width of the rod parallel to the facet is inversely proportional to the size of the facet: a broader streak is due to the small size of the facets. Under coherent illumination and no strain, thickness fringes are observed along the reciprocal rods with a period of one over the distance between the two opposite facets. Note that planar defects (like stacking-faults, antiphase or twin-boundaries) give also streaks perpendicular to the plane of the defect. $\frac{23}{2}$ Their associated fringes are no more correlated to the particle size. The direction of the rods can be used to determine the Miller indices $(h k l)$ of the facets.

A 3D view is often difficult to understand, even with interactive manipulation. We turned instead to a stereographic projection, which is conventionally used to express the geometry of crystals. The display is a scatter plot in which each normal vector is represented by a point on the stereographic projection.

A Python code has been developed to reduce the x-ray diffraction images of coordinates $\left(Q_{x}, Q_{y}\right.$ and $\left.Q_{z}\right)$ ( $Q_{x}$ being parallel to the x-ray beam) into $2 \mathrm{D}$ stereographic projections or pole figures $\left(\rho_{\text {pole }}, \theta_{\text {pole }}\right) . \rho_{\text {pole }}$ and $\theta_{\text {pole }}$ are the polar angles (i.e. the elevation and azimuth angles). The center of the sphere used to get the stereographic projection corresponds to the center of mass (COM) of the 3D diffraction pattern: $Q^{C O M}=\sum_{i} Q_{i} \cdot I_{i} / I_{\text {total }}$, where the sum runs over all the reciprocal space points $Q_{i}$. The upper or lower slice of hemisphere of radius $r$ and width $\Delta r$ is projected on a plane, corresponding to the equator plane of the sphere (see Fig. 4). $r$ verifies the following equation:

$$
r=\sqrt{\left(Q_{x}-Q_{x}^{C O M}\right)^{2}+\left(Q_{y}-Q_{y}^{C O M}\right)^{2}+\left(Q_{z}-Q_{z}^{C O M}\right)^{2}} .
$$

The azimuth angle, $\rho_{\text {pole }}$ can be calculated for each $\left(Q_{x}, Q_{y}\right.$ and $Q_{z}$ ) position: $\rho_{\text {pole }}=\arccos \left(\left(Q_{z}-Q_{z}^{C O M}\right) / r\right)$. It ranges from 0 to $90^{\circ}$. The $(u, v)$ normalised Euclidean metric coordinates of the stereographic projection of center of projection $(0,0,-r)$ (South pole) in the Wulff net follows:

$$
\begin{gathered}
u=\frac{Q_{x}-Q_{x}^{C O M}}{r+\left(Q_{z}-Q_{z}^{C O M}\right)} \\
v=\frac{Q_{y}-Q_{y}^{C O M}}{r+\left(Q_{z}-Q_{z}^{C O M}\right)} .
\end{gathered}
$$



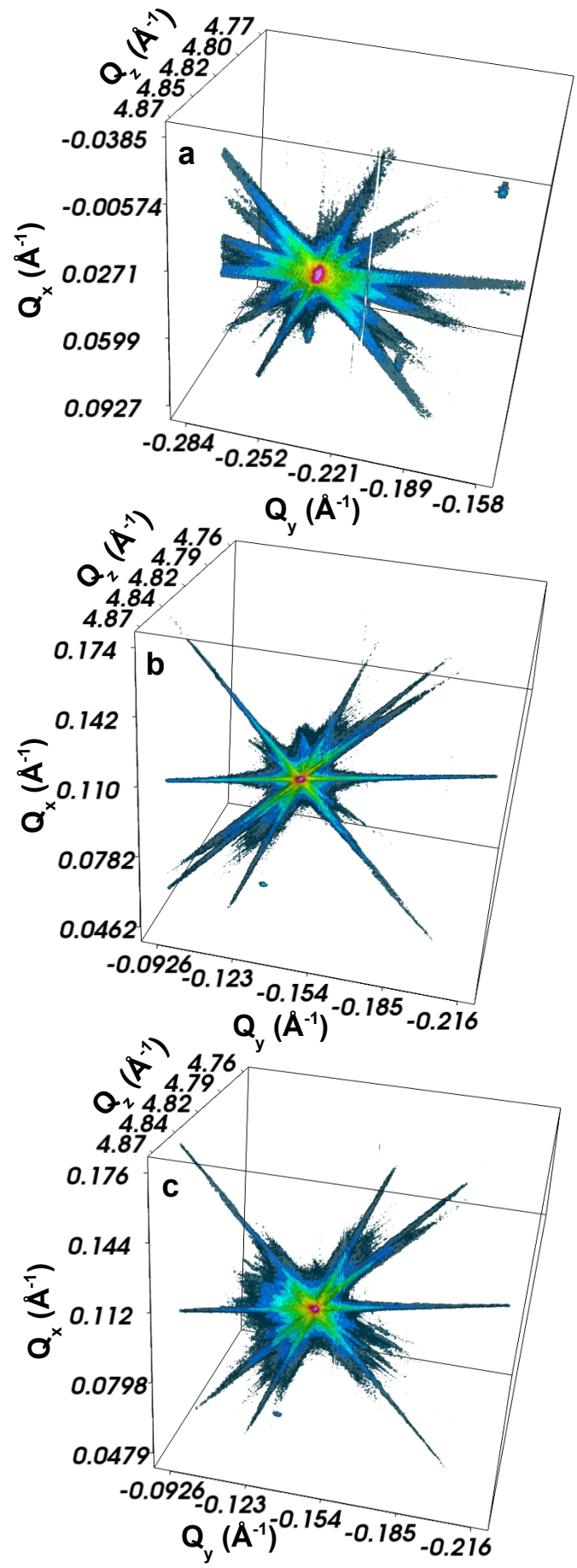

Fig. 3 Three-dimensional reciprocal space maps around the $004 \mathrm{GaN}$ Bragg reflection for two different single nanowires (a) and (b-c). Figures (b) and (c) have been measured on the same nanowire but at two different positions separated by $1 \mu \mathrm{m}$ along the wire axis.
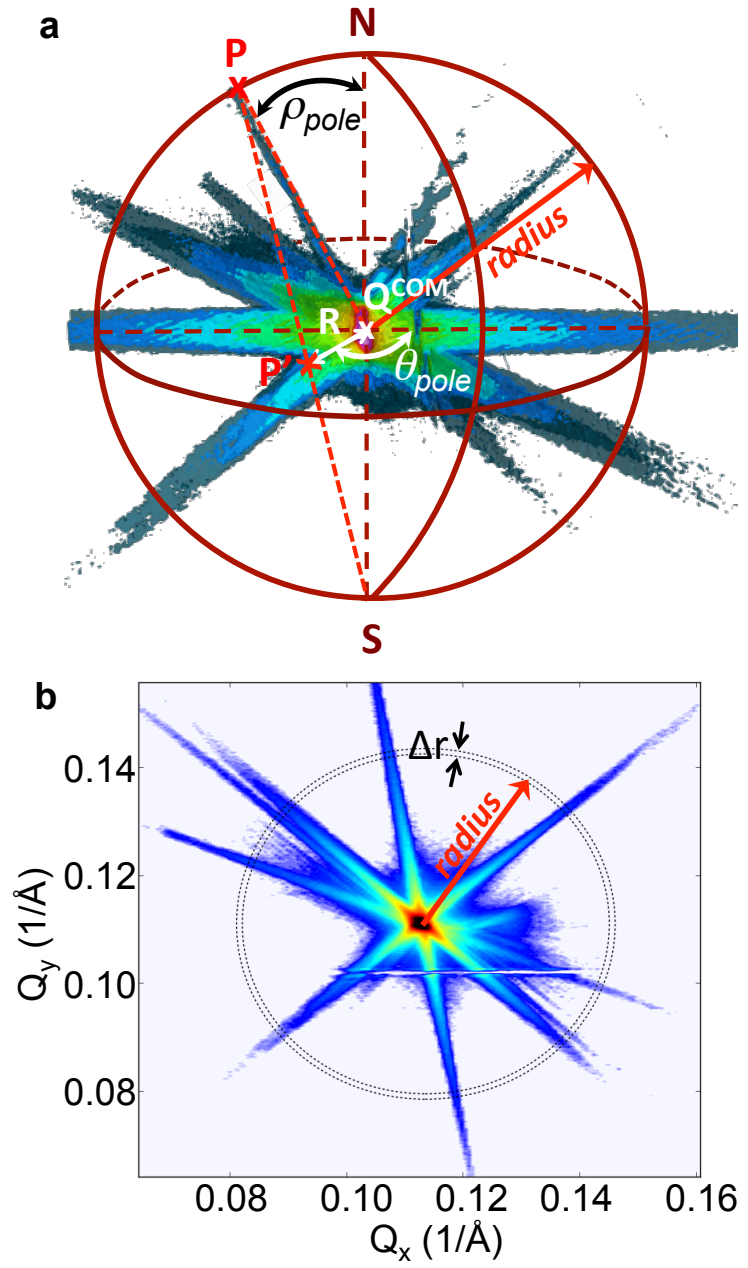

Fig. 4 (a) Illustration of the stereographic projection of one pole $(\mathrm{P})$ on the equator for Fig. 3(a). The projected pole $\mathrm{P}^{\prime}$ is the intersection of the line joining the South pole with the corresponding pole. The radius of the sphere, $r$ is indicated as well as the polar angles $(\rho, \theta)$ and the center of mass of the 3D scattered intensity $Q^{C O M}$. (b) In-plane $\left(Q_{x}, Q_{y}\right)$ reciprocal space map together with the intersection of the sphere of radius $r$ and width $\Delta r$.

$(u, v)$ is calculated for each $\left(Q_{x}, Q_{y}\right.$ and $\left.Q_{z}\right)$ pixel. $u, v$ and the intensity measured in the upper slice of hemisphere of radius $r$ and width $\Delta r$ are gridded (linear gridding) into a 2D matrix of size $300 \times 300$, for instance: $u_{\text {grid }}$, $v_{\text {grid }}$ with $I_{\text {grid }}$.

$\theta_{\text {pole }}$ is then calculated from $u_{\text {grid }}$ and $v_{\text {grid }}$ and follows: $\theta_{\text {pole }}=$ atan $2\left(v_{\text {grid }}, u_{\text {grid }}\right)$. It ranges from 0 to $360^{\circ}$. Generating a complete single pole figure from a set of $2 \mathrm{D}$ images is fully compatible with an online analysis of the data. It is done in less than $1 \mathrm{~min}$ (35 sec. for the conversion of a set of $2 \mathrm{D}$ images [300 images of $516 \times 516$ pixels] into $3 \mathrm{D}$ reciprocal space and $6 \mathrm{sec}$. for the conversion into a stereographic projection).

Figure 5 displays stereographic projections of the 3D reciprocal space maps shown in Fig. 3 for $r=0.028 \AA^{-1}$ and $\Delta r=0.001$ $\AA^{-1}$. Points of the pole figures verifying $\theta_{\text {pole }}=0$ correspond to points satisfying: $Q_{x}=Q_{x}^{C O M}$ and $Q_{y} \geq Q_{y}^{C O M}$. The intersection points of every streak with the hemisphere ("poles") are visualised on the pole figure (see black circles in Fig. 5). The symmetry 

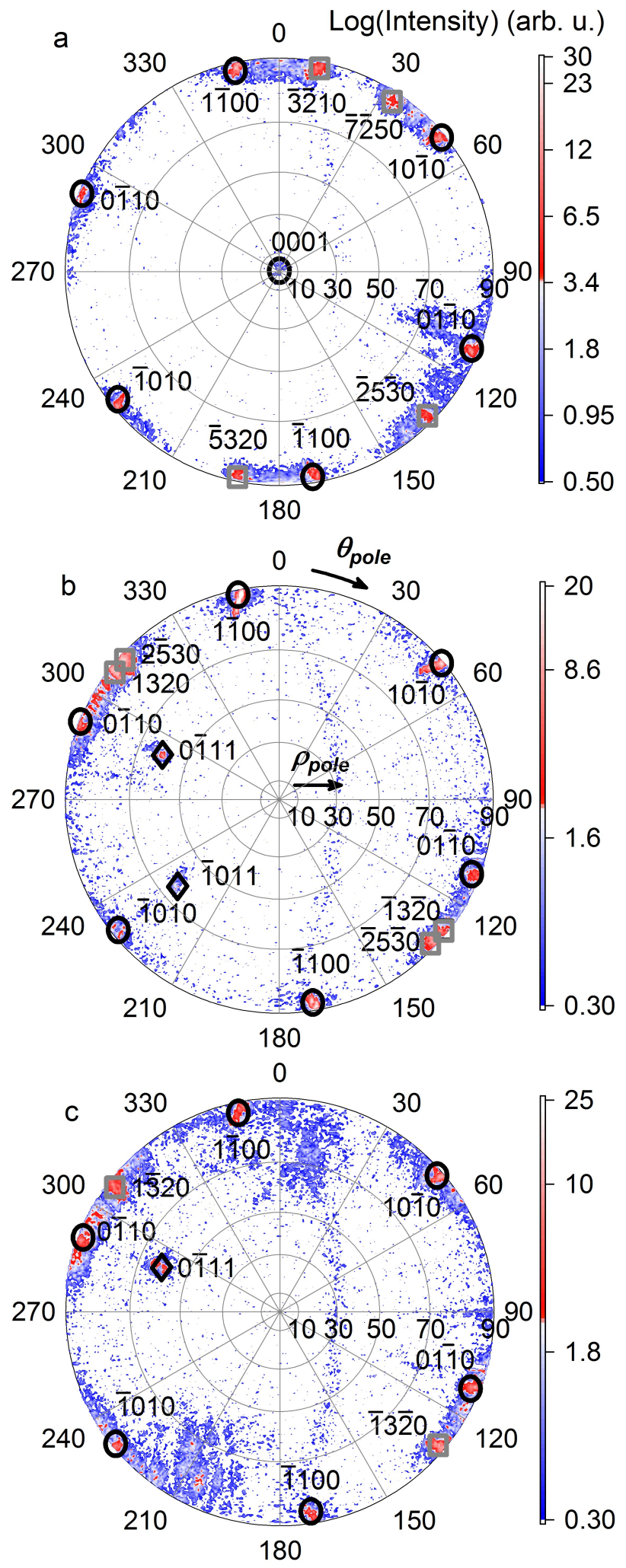

Fig. 5 ( $a, b$ and c) Stereographic projections of the 3D reciprocal space maps shown in Figs. 3 ( $a, b$ and $\mathrm{c}$ ) for $r=0.028 \AA^{-1}$ and $\Delta r=0.001 \AA^{-1}$. The black circles and diamonds as well as the grey squares indicate the position of intense peaks as well as their Miller index hkl.

and positions of the peaks arising from the streaks are related to the particular crystallographic structure of the studied material.
Every peak arises from a specific crystallographic axis, that can be in this way labelled with the corresponding $(h k l)$ Miller index of the facet or planar defect, knowing the crystallographic structure of the studied material (here, wurtzite structure). The method applied to experimental data allows indexing the facets of a single nanocrystal.

The pole figure indexing was performed using the MATLAB toolbox for texture analysis, MTEX [http://mtextoolbox.github.io/]. The only knowledge was that the central spot $\left(\rho_{\text {pole }}=0, \theta_{\text {pole }}=0\right.$ ) corresponds to the $\mathbf{0 0 0 1}$ pole as the 004 Bragg reflection has been measured in a coplanar geometry. All the pole figures or stereographic projections displayed in Fig. 5 show intense spots corresponding to lattice points of type 1100. They correspond to the lateral $m$-plane facets of the core-shell MQW GaN wires. One of these points has been arbitrarily fixed to $\mathbf{1} \mathbf{1 0 0}$ for all the pole figures corresponding to the wires. Other spots in the pole figures are identified. They correspond to (0111) and (1011) facets, which often form at the top of the core-shell MQW GaN wires. Interestingly, other lattice points (for instance, $\overline{\mathbf{3}} \overline{2} 10, \overline{\mathbf{5} 320}$ or $\overline{\mathbf{7}} \mathbf{2 5 0}$ ) are observed. They do not correspond to surface facets of the nanowires or to known surface defects: prismatic and pyramidal stacking faults are often lying in the (1100) and (1102) planes. To understand what can explain the supplementary lattice points observed in the pole figures, transmission electron microscopy (TEM) has been performed on similar samples (see Fig. 6). Interestingly, the transversal cross-sectional images taken along the $c$-zone axis show some bright lines starting from the intersection of the facets of the $\mathrm{GaN}$ wire and propagating through the core-shell InGaN/GaN multiple quantum wells. These defects may arise to permit relaxation at the $\mathrm{GaN}$ corners due to the different lattice mismatch between InGaN and GaN. These planar defects induced by the core-shell MQWs are foreseen to lead to 1D streaks in reciprocal space perpendicular to their surface planes. Their orientation in Fig. 6 explains the high-index spots, like $\overline{\mathbf{3 2}} \mathbf{2} \mathbf{5}, \overline{\mathbf{5} 320}$ or $\overline{\mathbf{7}} \mathbf{2 5 0}$, observed in the pole figures of Fig. 5 . The presence of well-defined spots implies that the defect planes are also well-defined. This measurement reveal new types of defects in core-shell multiple quantum wells nanowires as well as their location along the wire.

\subsection{Case of single Pt THH particles.}

The method can also measure and index facets of complex and randomly oriented 3D nanoparticles. Figure 7 displays the complex 3D reciprocal space map of the $\mathbf{1 1 1}$ Bragg reflection of an individual Pt particle of $350 \mathrm{~nm}$ diameter as a function of reciprocal space coordinates $Q_{x}, Q_{y}$ and $Q_{z}$. The truncation rods or streaks correspond to the different facets of the particle, each streak being perpendicular to each facet. The sharp and welldefined directions of the streaks allows one to index the Miller indexes of the facets. Figure 8 (a) displays a stereographic projection of the 3D reciprocal space map shown in Fig. 7 (upper hemisphere: $Q_{z}>Q_{z}^{C O M}$ ) for $r=0.037 \AA^{-1}$ and $\Delta r=0.0005 \AA^{-1}$. The measured reflection was the symmetrical 111 Pt Bragg reflection, meaning that the $Q_{z}$ direction is approximately along the [111] 


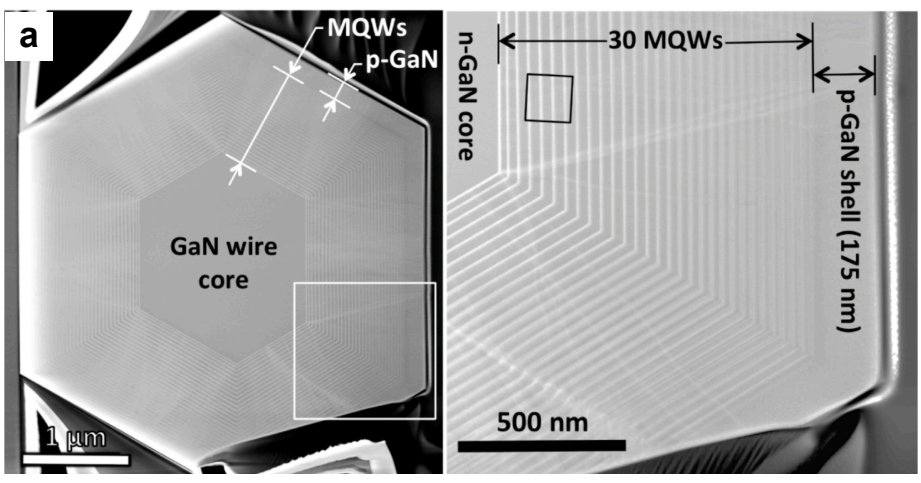

Fig. 6 (a) Transversal cross-sectional STEM-HAADF images taken along the $c$-zone axis show the core-shell structure on $m$-plane hexagonal facets (wire slice prepared by FIB technique). (b) Increased magnification of the TEM image. Adapted from Ref. ${ }^{24}$

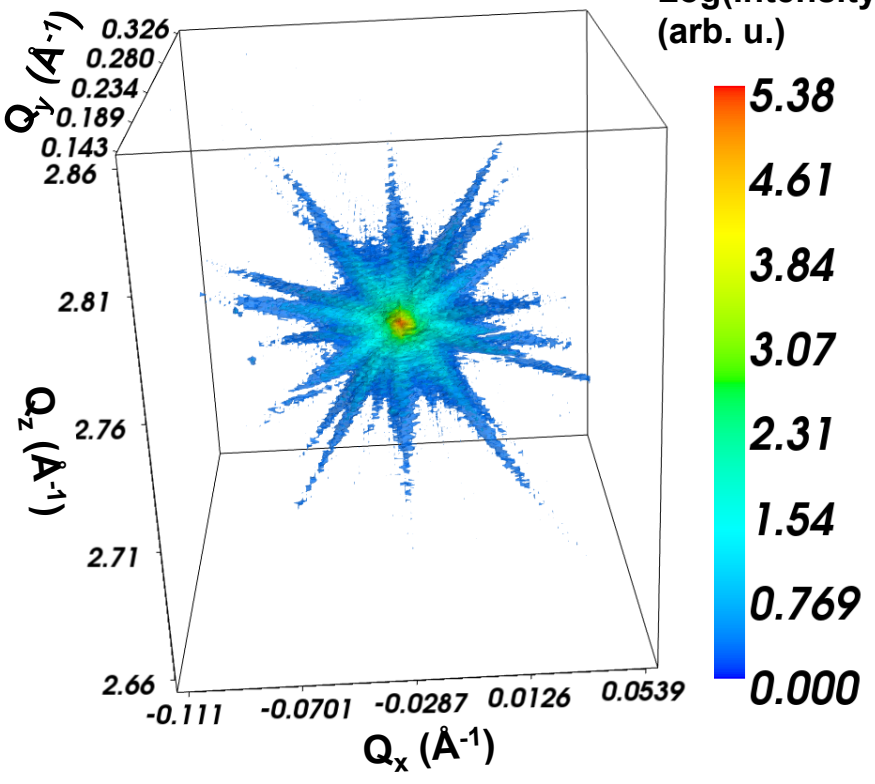

Fig. 7 Three-dimensional reciprocal space map of the $\mathbf{1 1 1}$ Bragg reflection of an individual Pt particle of 350-nm diameter.

direction (see the black cross at the center of the pole figure in Fig. 8(a)). Note that the green circles indicate the location of the 201-type poles. The circles are centered on the position of the 111 pole, which is not often at the center of the pole figures, due to small misorientations of the $\{111\}$ planes of the Pt particles with respect to the sample surface. It is known that most electrochemically synthesised Pt particles can be bound by $\{h k 0\}$ ( $\{210\}$, $\{310\},\{520\}$ and/or $\{730\})$ facets. They are known as tetrahexahedral (THH) particles. ${ }^{[16}$ Here, the best fit has been obtained by a set of 12 poles of 201 type, corresponding to 12 \{201\} facets for $Q_{z}>Q_{z}^{C O M}$ (accuracy of $\pm 0.5^{\circ}$ ). Figures 8 (b-c) display the stereographic projection of the 3D reciprocal space map (upper hemisphere: $Q_{z}>Q_{z}^{C O M}$ ) of two different individual sub-surface $\mathrm{Cu}$ alloyed THH Pt particles. In these two cases, poles of 201 type are observed in addition to others. In Fig. 8(b), the pole (indicated by a red circle), which is not positioned on one of the green cir- 
of the particle shows at least two types of facets (a majority of $\{201\}$ facets and at least one $\{205\}$ facet). In Fig. 8(c), the 10 poles of 201 type are observed. There is an additional pole corresponding to the intersection of a [11̄î] streak with the sphere. The streak comes either from a $\{111\}$ facet or a $\{111\}$ stackingfault. Stacking-faults are fairly common in $f c c$ metals, like Pt, and usually occur in $\{111\}$ crystallographic planes. Here, additional measurements (like Bragg coherent x-ray diffraction) are needed to discriminate if the signal is from a facet or a fault. Two additional poles (not positioned on the green circles) are not of 201type poles and can be indexed by 136-type poles. These poles are not of $\boldsymbol{h} \boldsymbol{k} \mathbf{0}$-type. They are thus uncommon in THH particles. Note that the same conclusions could be drawn for 3D reciprocal space maps from other directions. Another set of data centered on the position of the $\mathbf{1 0 0}$ pole and for another particle is presented in Figure S1 of the Supplementary Material. \{201\}-type facets are well observed.The technique demonstrates its prowess to determine the crystallographic orientation of facets and planar defects inside nanostructures. This technique can also be extended to characterise highly faceted and complex crystalline nanoparticles and study how their faceting evolves during in situ and operando reactions. This will enable a systematic investigation of the effect of surface structure and refaceting during catalytic reactions.

\section{Conclusions}

By using a fast readout, photon-counting two-dimensional pixel detector and a nano-focused x-ray beam, we were able to characterise the crystallographic planes at the surface of a single nanostructure, as well as planar defects. A Python routine allows for the conversion of the full 3D data into pole figures. To illustrate the possibilities, we have mapped a core-shell multiple quantum well nanowire as well as highly faceted nanoparticles. We have demonstrated that the facets of the nanocrystals can be extracted from their 3D reciprocal space maps. In the case where two or more non-parallel facets are present, it is possible to identify the orientation of the crystal. The method also reveals new types of defects in core-shell multiple quantum wells nanowires as well as their location along the wire. It also reveals uncommon facets in THH Pt particles. The described approach is a promising tool for structural characterisation of nanostructures, and due to the penetration power of hard $\mathrm{x}$-rays, it could be especially useful for embedded structures where techniques like scanning electron microscopy or transmission electron microscopy are unable to probe. The non-invasive nature of CXDI as well as the pole figure analysis is particularly well adapted to study nanoscale materials during in situ and operando experiments, like catalysis, thermal treatment or corrosion. It opens new ways to study nanoscale materials during operation allowing facet and planar defects identification to be performed.

\section{Acknowledgments}

The experiments were performed on beamline ID01 at the European Synchrotron Radiation Facility (ESRF), Grenoble, France. We thank the ID01 beamline staff for excellent support during the experiment. Financial support for this work by ANR Charline (ANR-16-CE07-0028-01) is gratefully acknowledged.

\section{Conflict of interest}

There are no conflicts to declare.

\section{Author contributions}

M.I.R and S.L. designed the project. M.I.R, S.F., J.P.H. and L.G. performed the synchrotron measurements. M.I.R., S.F. and J.C. analyzed the synchrotron data. J.E. and L.G. prepared the samples. All contributed to data discussion and to writing the manuscript.

\section{References}

1 L. Y. Chang, A. S. Barnard, L. C. Gontard and R. E. DuninBorkowski, Nano Letters, 2010, 10, 3073-3076.

2 C. Pecharromán, J. Pérez-Juste, G. Mata-Osoro, L. M. LizMarzán and P. Mulvaney, Phys. Rev. B, 2008, 77, 035418.

3 W. Gibbs, Transactions of the Connecticut Academy, 1875, 108248.

4 M. C. Scott, C.-C. Chen, M. Mecklenburg, C. Zhu, R. Xu, P. Ercius, U. Dahmen, B. C. Regan and J. Miao, Nature, 2012, 483, 444-447.

5 S. Van Aert, K. J. Batenburg, M. D. Rossell, R. Erni and G. Van Tendeloo, Nature, 2011, 470, 374-377.

6 B. Goris, A. De Backer, S. Van Aert, S. Gómez-Grana, L. M. Liz-Marzán, G. Van Tendeloo and S. Bals, Nano Letters, 2013, 13, 4236-4241.

7 A. B. Shah, S. T. Sivapalan, B. M. DeVetter, T. K. Yang, J. Wen, R. Bhargava, C. J. Murphy and J.-M. Zuo, Nano Letters, 2013, 13, 1840-1846.

8 P. A. Midgley and R. E. Dunin-Borkowski, Nat Mater, 2009, 8, 271-280.

9 I. Robinson and R. Harder, Nat Mater, 2009, 8, 291-298.

10 B. L. Adams, S. I. Wright and K. Kunze, Metallurgical Transactions A, 1993, 24, 819-831.

11 F. Hofmann, N. W. Phillips, R. J. Harder, W. Liu, J. N. Clark, I. K. Robinson and B. Abbey, J Synchrotron Rad, J Synchrotron Radiat, 2017, 24, 1048-1055.

12 T. Kuykendall, P. Ulrich, S. Aloni and P. Yang, Nat Mater, 2007, 6, 951-956.

13 B. Jiang, C. Zhang, X. Wang, F. Xue, M. J. Park, J. S. Kwak and M. Xiao, Optics Express, 2012, 20, 13478.

14 R. Koester, J.-S. Hwang, D. Salomon, X. Chen, C. Bougerol, J.-P. Barnes, D. L. S. Dang, L. Rigutti, A. de Luna Bugallo, G. Jacopin, M. Tchernycheva, C. Durand and J. Eymery, Nano Letters, 2011, 11, 4839-4845.

15 A. Messanvi, H. Zhang, V. Neplokh, F. H. Julien, F. Bayle, M. Foldyna, C. Bougerol, E. Gautier, A. Babichev, C. Durand, J. Eymery and M. Tchernycheva, ACS Applied Materials \& Interfaces, 2015, 7, 21898-21906.

16 N. Tian, Z.-Y. Zhou, S.-G. Sun, Y. Ding and Z. Lin Wang, Science, 2007, 316, 732-735.

17 J. Xiao, S. Liu, N. Tian, Z.-Y. Zhou, H.-X. Liu, B.-B. Xu and S.G. Sun, Journal of the American Chemical Society, 2013, 135, 18754-18757.

18 M. T. M. Koper, Nanoscale, 2011, 3, 2054. 
19 R. Koester, J. S. Hwang, C. Durand, D. L. S. Dang and J. Eymery, Nanotechnology, 2010, 21, 015602.

20 A. A. Proussevitch and D. L. Sahagian, Computers and Geosciences, 2001, 27, $441-454$.

21 C. Ponchut, J. M. Rigal, J. Clément, E. Papillon, A. Homs and S. Petitdemange, J. Inst., 2011, 6, C01069.

22 D. Kriegner, E. Wintersberger and J. Stangl, Journal of Applied Crystallography, 2013, 46, 1162-1170.
23 P. Ehrhart, H. Trinkaus and B. C. Larson, Phys. Rev. B, 1982, 25, 834-848.

24 H. Zhang, X. Dai, N. Guan, A. Messanvi, V. Neplokh, V. Piazza, M. Vallo, C. Bougerol, F. H. Julien, A. Babichev, N. Cavassilas, M. Bescond, F. Michelini, M. Foldyna, E. Gautier, C. Durand, J. Eymery and M. Tchernycheva, ACS Applied Materials \& Interfaces, 2016, 8, 26198-26206. 
Novel approach based on nano-focused coherent Bragg $\mathrm{x}$-ray imaging to characterise the crystallographic planes at the surface of single nanostructures and planar defects

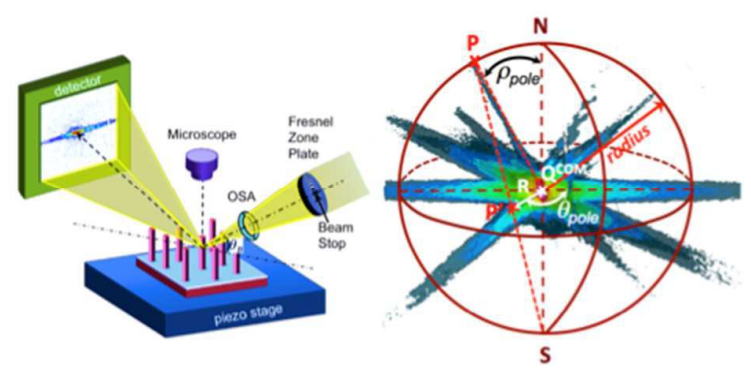

